\title{
Canon littéraire et enseignement de la littérature française au Canada anglais: L'exemple des femmes auteurs
}

\section{RACHEL SAUVÉ \\ Université du Nouveau-Brunswick}

\section{RÉSUMÉ}

Cet article aborde la problématique de l'enseignement de la littérature dite classique (pour notre propos, la littérature française du XIXe siècle) dans les universités anglophones au Canada, et propose de nouveaux critères pour la sélection des cuvres à enseigner: ancrage d'une œuvre dans sa propre époque, rapports de cette œuvre à notre époque, et rapports intertextuels. Des remarques sur les théories de la formation du canon littéraire, qui désigne ici le corpus des æuvres enseignées, sont suivies d'observations sur la situation dans les universités canadiennes, en prenant l'exemple de la littérature française du XIXe siècle. Le cas des œuvres de femmes, emblématique dans l'histoire de la formation du canon, est ensuite cité en exemple, avec à l'appui une brève étude des tendances actuelles dans la mise en ligne des textes de femmes, qui confirme le maintien des critères traditionnels sur Internet. Par ailleurs, les nouvelles technologies, en favorisant une réflexion sur le format même des cours de littérature, un format plus

Remerciements aux collègues qui m'ont gentiment communiqué leurs plans de cours: Janice Best, Peter Edwards, Dominique Laporte, Malcolm McGoldrick, Tony Purdy. 
éloigné des périodisations et de la notion d'auteur, et plus favorable à une mise en réseaux intertextuels des textes littéraires, sont à même de susciter des changements substantiels dans les programmes et la sélection des œuvres enseignées.

\section{ABSTRACT}

This article deals with the teaching of French classical literature in Canadian universities, and promotes new criteria in the selection of works to be taught: relevance of the work to its own times, its relevance for our times, and intertextual relevance. Preliminary remarks on theories of canon formation are followed by comments on the situation in Anglophone Canadian universities, supported by data on 19th Century French Literature courses. The example of women's writings, which is highly representative of the canon formation process, is developed and includes a survey of the current trends in the accessibility of women's writings on the Web, which confirms the continuing precedence of traditional criteria in the digitalization of literary texts. Nevertheless, the new technologies allow for a rethinking of the course format itself, which could be renewed by avoiding traditional period and author patterns, and by favoring the intertextual networking of literary texts, thus bringing about substantial changes in Canadian literature programs.

À l'heure où les sciences humaines semblent menacées, en Amérique du Nord, par le discours néo-libéral, il convient de s'interroger sur le rôle des études littéraires dans l'éducation postsecondaire au Canada anglais. Ce questionnement soulève un débat, que certains désignent, à l'instar de Ross (1996), comme les «canon wars» (p. 432) opposant ainsi les tenants des études culturelles et ceux des études strictement littéraires. Ces derniers, qui empruntent, selon les termes de Fleming (2000), au paradigme de la sagesse plutôt qu'à celui du savoir, préconisent le maintien d'un canon littéraire fondé sur la valeur esthétique, cette valeur reposant sur des critères tels que «la maîtrise du langage figuratif, l'originalité, la connaissance, le savoir,» que fait valoir Bloom (1994) dans The Western Canon (p. 29'); d'autres, 
comme Said (1999), prônent la découverte de réseaux dialogiques entre des textes émergeant respectivement du centre et des marges:

The reading and interpretation of classics must not stop at veneration and respectful appreciation but needs to go on from there to uncover the enormously interesting networks of relations between canonical and noncanonical writing. (p. 3)

Il appert toutefois que, malgré les progrès importants des études culturelles au Canada et aux États-Unis, les critères traditionnels de valorisation sont toujours à l'œuvre dans l'institution. Si, comme l'affirme Angenot (1997), le ressentiment a parfois fait glorifier la médiocrité, allant parfois jusqu'à «chercher à briser les instruments de la valorisation» (p. 14), souvent aussi la circularité des critères esthétiques et un présupposé d'universalisme ont causé l'exclusion de textes littéraires qui présentent des variations par rapport à des normes génériques et esthétiques pré-définies. Dans ce contexte, l'enseignement de la littérature française dans les universités anglophones pose des problèmes particuliers, pour lesquels je proposerai ici quelques pistes des réflexion. Pour étayer mon propos, je prendrai l'exemple emblématique des textes des femmes du XIXe siècle, qui constituent un cas incontournable d'exclusion. En effet, la production littéraire des femmes au XIXe siècle est remarquable et son exclusion quasi intégrale du corpus des cuvres enseignées l'est tout autant. La tendance actuelle dans la mise en ligne des textes du XIXe siècle semble aller dans le sens de cette exclusion, ce qui illustre l'importance, voire l'urgence, d'une révision des critères d'inclusion des cuvres littéraires dans l'enseignement et dans Internet.

\section{LA REPRÉSENTATIVITÉ}

On a vu pour certaines époques (le XVIIIle et le XXe siècle français), certaines littératures nationales (québécoise, africaine et antillaise) et divers genres littéraires (la paralittérature, la science-fiction), un véritable élargissement du canon littéraire permettant l'inclusion des textes de femmes. On ne peut que se réjouir de ces avancées, même si les textes des femmes sont encore confinés dans des cours «spécialisés»: cela est 
probablement souhaitable, du moins à moyen terme, puisque le contexte de production et de réception de ces textes requiert un cadre d'analyse particulier. Mais cette ouverture laisse néanmoins aux professeurs de littérature dite classique (de la Renaissance au XIXe siècle) des universités anglophones canadiennes la tâche d'enseigner le canon alors que le nombre des cours de littérature est réduit pour laisser une place nécessaire aux cours de langue. D'où un malaise. D'une part le canon, que l'on pourrait désigner sommairement, pour paraphraser Barthes (1984), comme «ce qui s'enseigne» (p. 49), représente l'expression de tout ce qui est honni de nos jours: incorrectitude politique, racisme, sexisme et classisme. De plus les étudiants dont le français n'est pas la première langue ne la maîtrisent pas suffisamment pour une lecture fructueuse de ces textes. Mais d'autre part le canon comporte des textes et des auteurs incontournables, non seulement pour leur valeur littéraire mais en tant qu'exemples des efforts soutenus de l'institution pour maintenir leur statut. ${ }^{2}$ À cet égard, le postulat de Jacques Dubois (1978), malgré sa terminologie un peu dépassée, est toujours valable: «La littérature est une institution, à la fois comme organisation autonome, comme système socialisateur et comme appareil idéologique» (p. 34). En tant que système socialisateur, c'est à l'enseignement que l'institution littéraire confie la tâche de la soutenir: on enseigne ce que l'on a soimême étudié, ce qui entraîne une fermeture du corpus enseigné.

Cependant, voulant enseigner la littérature, et non pas l'histoire ou la civilisation, où trouver les heures nécessaires à l'explication du contexte indispensable pour une lecture un tant soit peu éclairée des cuvres classiques? Doit-on simplement survoler, en laissant tomber ici et là quelque morceau d'anthologie, un XIXe siècle qui exige, par sa richesse, qu'on y plonge? Et comment mettre à profit les ressources d'Internet, dont l'avènement constitue un moment crucial pour le renouveau de nos cours, alors que l'on observe actuellement, dans la mise en ligne des textes littéraires, le maintien des critères d'inclusion et d'exclusion traditionnels?

Notons sans attendre que la valeur littéraire n'étant pas un critère explicitement avoué dans la sélection des œuvres à enseigner, elle n'est 
pas nécessairement au coeur de cette réflexion. Jonathan Culler (1997) précise:

Each teacher does not pick what he or she thinks are the ten greatest works of world literature but, rather, selects works that are representative of something: perhaps a literary form or a period of literary history. (p. 50)

«Représentatif,» "période,» voilà bien les deux mots-clés: la périodisation constitue le découpage ordinaire, quoique non exclusif, des cours universitaires au Canada anglophone, et la représentativité des œuvres est donnée comme un critère déterminant, comme en témoigne un échantillon (Tableau 1) des descripteurs des cours sur le dixneuvième siècle offerts dans dix universités canadiennes anglophones à travers le Canada en 2000-2001. ${ }^{3}$

À l'exception des descripteurs 1 et 9 , qui proposent une vision plus «culturelle» de l'époque romantique, ${ }^{4}$ les cours offerts reposent clairement sur la périodisation traditionnelle et sur le découpage de l'époque en mouvements littéraires. On observe aussi l'emploi fréquent du mot «représentatif» et de ses substituts ("caractéristiques,» «majeurs») pour désigner les cuvres et les auteurs, dont on peut aussi établir une liste fermée à partir des descripteurs et des plans de cours que m'ont gracieusement transmis plusieurs professeurs responsables de ces cours, liste qui peut se diviser en deux sous-groupes: celui des auteurs cités fréquemment (Hugo, Chateaubriand, Lamartine, Sand, Vigny, Balzac, Flaubert, Baudelaire, Maupassant, Zola, Rimbaud, Verlaine, Mallarmé, Mme de Staël, Musset) et celui des auteurs cités une fois, dont on observe d'emblée le caractère plus hétéroclite: DesbordesValmore, Rostand, Stendhal, Sainte-Beuve, Mérimée, Constant, Gautier, Nerval, Banville, Leconte de Lisle, Laforgue. Rien là que de prévisible ${ }^{5}$ (on a ici de quoi faire la table des matières d'une anthologie), mais c'est tout le paradoxe de cette soi-disant représentativité des Hugo, Rimbaud et Balzac que je souhaite mettre en lumière, surtout quand on prétend, comme c'est souvent le cas, restituer le contexte de l'époque. En effet, la plupart des auteurs «majeurs» dont les cuvres constituent l'essentiel du corpus des œuvres enseignées actuellement sont plutôt des exceptions que des auteurs typiques et si leurs œuvres représentent bien chacun des 


\section{Titre du cours}

1. Introduction à la littérature française du XIXe siècle

2. Littérature du XIXe siècle

3. Barricades, bordels et champs de bataille

4. Le 19e siècle : débuts

\section{Descripteur}

L'objectif de ce cours sera double: 1) donner un survol de l'histoire littéraire du siècle en fonction des grands mouvements qui le caractérisent: du romantisme au symbolisme, du réalisme au naturalisme; 2) étudier les enjeux de la représentation littéraire en analysant les rapports avec la réalité sociale.

Introduction à la littérature du XIXe siècle : romantisme, symbolisme, réalisme, naturalisme. Étude d'oeuvres représentatives de l'époque.

Chansons, pamphlets révolutionnaires, tableaux, textes littéraires, lettres et manifestes nous permettront d'examiner comment la société française évolue de la Révolution vers la démocratie. On étudiera tout

spécialement les lieux de rencontre, dont la nature influence le type des récits qui contribuent à transformer la société.

Romantisme et réalisme. Auteurs choisis parmi Chateaubriand,

Desbordes-Valmore, Hugo, Lamartine, Mérimée, Mme de Staël, Musset, Stendhal, Vigny.

5. Le Romantisme II 
Tableau 1 (cont.)

\section{Titre du cours}

6. La Poésie de Baudelaire à Valéry

\section{Descripteur}

Étude et analyse de textes choisis dans les oeuvres poétiques majeures de 1850 à 1920. Parmi les poètes étudiés : Nerval, Baudelaire, Banville, Leconte de Lisle, Rimbaud, Verlaine, Mallarmé, Laforgue, Apollinaire, Valéry et quelques autres. Nous choisirons des thèmes communs abordés par plusieurs poètes et nous approfondirons l'évolution prosodique.

7. Roman et nouvelle français du dix-neuvième siècle

Étude des principaux romanciers romantiques, réalistes et naturalistes: un choix de Chateaubriand, Constant, Balzac, Sand, Flaubert, Zola, Maupassant.

8. Le romantisme, le réalisme et la littérature du $19 \mathrm{e}$ siècle
Ce cours se propose de présenter une vue d'ensemble du $19 \mathrm{e}$ siècle et d'analyser, à partir d'oeuvres particulièrement significatives, les principaux mouvements littéraires de l'époque en les situant dans leur contexte artistique et social. Le romantisme, le réalisme, le naturalisme et le symbolisme expriment, à leur manière, une époque en pleine effervescence, marquée par de grands événements politiques (l'épopée napoléonienne, la Commune de Paris) et par des modes d'expression artistique originaux et variés (drame romantique, photographie, peinture impressionniste, etc.). Les œuvres, choisies pour leur représentativité, seront à la fois étudiées pour leur valeur littéraire et replacées dans leur contexte socio-culturel. Chaque fois que cela sera possible, on fera appel à des documents audio-visuels pour illustrer les aspects novateurs de ce siècle qui ouvre sur la modernité. 


\section{Titre du cours}

9. La révolution romantique

10. Aspects du romantisme

\section{Descripteur}

Romanticism is viewed primarily as a rebellious and creative force which greatly contributed to the reshaping of traditional society. The origins, main themes and trends of the movement are studied with and attempt to show Romanticism as a European movement, the impact of which was felt in fields beyond the boundaries of literature.

Étude du mouvement romantique à travers les oeuvres les plus représentatives des auteurs les plus connus : Chateaubriand, Hugo, Lamartine, Sand, Sainte-Beuve, Vigny et les membres du petit Cénacle.

Note: Ces descripteurs ont été recueillis sur Internet ; dans plusieurs cas (vérification faite auprès des instructeurs), ils sont modifiés dans le plan de cours tel qu'établi de façon définitive. De plus, certaines institutions offrent plutôt des cours portant sur des genres littéraires : fantastique, essai, littérature pour la jeunesse, théâtre, qui peuvent inclure des oeuvres du XIXe siècle. 
mouvements littéraires clairement identifiés (romantisme, réalisme, naturalisme, symbolisme), c'est que ces mouvements ont été définis en fonction de leurs œuvres. Il ressort donc que bon nombre d'entre nous entérinent ainsi le canon tel que défini par Miller (1990), c'est-à-dire:

un processus de sélection par lequel les pratiques d'écriture et de lecture, de même que les préférences d'une époque culturelle donnée sont effacées, oubliées et réécrites comme une histoire littéraire transcendante (p. 335).

Certes une tendance nouvelle se dessine, qui est déjà mise en pratique aux $2 \mathrm{e}$ et $3 \mathrm{e}$ cycles. Des cours sur les littératures minoritaires, sur le poème en prose, le fantastique, la nouvelle, l'essai, décloisonnent les siècles et permettent de rétablir la dimension dialogique des textes littéraires. Ces cours se définissent moins en fonction des auteurs que des textes, ce qui favorise la réflexion théorique, en particulier sur les zones de transition des mouvements et des genres. Mais encore une fois, les apparences peuvent être trompeuses, comme l'illustrent les descripteurs de deux cours offerts aux études supérieures, accompagnés des noms des auteurs au programme.

Paradoxalement, c'est le cours qui se présente apparemment comme le plus inspiré par les «études culturelles», le second, qui propose le choix d'auteurs le plus conventionnel. On ne peut que constater combien le poids de la tradition limite encore notre accès à une diversité existante mais méconnue. Un prolongement de cette enquête serait d'analyser les descriptions des postes offerts en littérature par les départements d'études françaises à travers le pays, et qui confirmerait que la périodisation domine là aussi. Mais revenons plutôt au baccalauréat.

\section{POUR UNE ARCHÉOLOGIE LITTÉRAIRE}

Le problème principal est que le nombre des auteurs canoniques du XIXe siècle est écrasant, et laisse peu d'espace, dans le cadre de cours de trois mois, aux auteurs dits «mineurs» ou tout simplement minorisés. Coste (1989), dans son manifeste pour une histoire littéraire négative, parle des objets de non-valeur, et dit bien que «la dévaluation et la détérioration ne sont ni spontanées ni gratuites, même quand elles 
1. Le poème en prose au XIXe siècle

On se demandera si le poème en prose peut au mieux se définir par le refus des contraintes génériques, s'il n'est en somme qu'un refuge pour l'inclassable, ou si des oeuvres étudiées se dégage un consensus suffisant pour permettre d'établir un certificat de genre en bonne et due forme.

\section{Nous examinerons dans ce cours les} différentes façons dont Paris fut textualisé au XIXe siècle; c'est-à-dire, comment Paris fut construit dans et par divers textes, comment il servit de sujet, de topos, de trope. Nous considérons [sic] comment certains écrivains s'approprièrent la ville de Paris pour en faire un espace discursif particulier dans lequel ils créaient, célébraient, contestaient, et transformaient des valeurs et des sens. Comme supplément aux textes que nous lirons, nous consulterons aussi diverses appropriations et constructions visuelles de Paris, surtout pour la deuxième moitié du siècle.
Bertrand, Baudelaire, Cros,

Rimbaud, Frénaud, Huysmans, Jacob, Mallarmé

\section{Balzac, Baudelaire, Flaubert,} Rimbaud, Zola 
prennent l'allure de l'abandon, de l'évanouissement, de la disparition silencieuse» (p. 19). La première conséquence de ces absences est que nous perdons de vue la valeur attribuée aux œuvres quand elles ont paru, et donc la notion de leur ancrage dans la conjoncture d'où elles émergent. Dès lors toute évaluation de la représentativité des œuvres semble précaire, parce que nous avons égaré la plus grande part de la production littéraire, du moins en ce qui concerne le XIXe siècle. Ne nageons-nous pas en plein paradoxe? En quoi et de quoi Flaubert et Hugo sont-il représentatifs? Et comment apprécier leur transcendance lorsqu'on n'est pas en mesure, comme le dit Mozet (1992), «d'apprécier de quelle gangue idéologique ils sont parvenus à s'extraire» (p. 4)?

La littérature exclue par l'institution est marquée par l'anonymat où ont sombré ses auteurs. Or nous participons de la croyance implicite que la source d'un grand texte se trouve dans l'esprit unique, génial, démesuré, bref transcendant, d'un individu exceptionnellement doué. Il s'agit d'une idéalisation de la fonction-auteur, d'une théorie d'autogenèse tenace qui nous empêche, soutient Clark (1994), de considérer l'origine de l'écriture comme «une source qui ne consiste ni dans une intentionnalité, ni dans les pulsions somatiques de l'inconscient, ni dans les structures et les contraintes du discours, mais dans la collaboration passagère et impondérable de tous ces facteurs» (p. 24). La critique littéraire, n'ayant pas su mettre en cause les bases épistémologiques de ses analyses, continue de prendre comme présupposée la fonction-auteur, qui renvoie, maintient-on, au «lieu originaire de l'écriture» (Foucault, 1969, p. 85). Que le point de vue privilégié pour l'analyse soit le texte, le lecteur, l'auteur, son inconscient, son habitus ou ses manuscrits, il semble bien que l'on cherche toujours le grand homme dans l'œuvre. Le postulat de la critique traditionnelle est paraphrasé par Eagleton (1983) dans ces termes ironiques: «Great literature is the product of Great men, and its value lies chiefly in allowing us intimate access to their souls» (pp. 47-48). Ce postulat n'a encore été véritablement remis en question ni par la critique structuraliste ni par l'herméneutique, ni par les études génétiques ou psychocritiques. De plus, comme en fait foi le passage suivant, tiré de 
l'article récent de Fleming (2000), il trouve un écho toujours vivace dans la promotion des études littéraires traditionnelles:

Literary studies for the new millennium have no value, save insofar as that is based on the value for the individual reader: and the path to finding this value cannot be predicted, schematized, nor institutionalized. (p. 475)

\section{L'EXEMPLE DES FEMMES AUTEURS}

Les descripteurs donnés en exemple plus haut montrent bien que les œuvres des femmes font piètre figure dans les cours de littérature au Canada anglophone. Leur exclusion du canon, et donc de ce qui s'enseigne, nous prive de leurs textes, qui enrichiraient nos corpus tant sur le plan de la contextualisation des cuvres que sur celui de la représentation des femmes dans la littérature, et renouvelleraient notre enseignement de la littérature. Cela dit, bien que les œuvres des femmes aient fait les frais de l'importance hyperbolique attribuée à la fonctionauteur dans la valorisation des œuvres littéraires, d'autres champs oblitérés par le même procès de dévalorisation pourraient aussi être évoqués: littératures non-hexagonales, littérature populaire, policière, de science-fiction, etc. $\dot{A}$ chacun de substituer le corpus de son choix aux remarques qui suivent, et qui proposent d'illustrer les processus d'exclusion.

En France au XIXe siècle, les femmes sont très nombreuses à écrire (2000, selon l'inventaire de Slama, 1992) et c'est le travail d'exclusion ayant pris place ultérieurement qui explique la disparition de leurs œuvres (Sauvé, 2000). Le cas de Delphine de Girardin est certainement, à cet égard, exemplaire: prolifique, célèbre, presque vénérée par les assidus de son salon, elle fut poète, tragédienne, romancière, chroniqueure. La notice posthume qu'écrivit Théophile Gautier pour ses Euvres complètes, en 1858, rend justice au statut qu'elle avait à sa mort (en 1855), statut comparable à celui de Balzac, sur qui Gautier écrivit aussi une notice posthume ces années-là. À propos de Girardin, Gautier (1857) commence ainsi sa notice: 
Les CEuvres complètes de madame Émile de Girardin n'avaient pas encore été réunies dans un format digne d'elles. Désormais les amoureux de ce charmant esprit ne seront plus obligés de le chercher à travers des volumes disparates, peu faits pour les rayons d'une bibliothèque sérieuse. (p. i)

Il avait bien tort, car seules les Chroniques parisiennes, rédigées, ironiquement, sous un pseudonyme masculin, sont aujourd'hui disponibles. Gautier a lui-même initié une fragmentation de l'auteur qui a contribué, en définitive, à sa disparition. Selon lui, il y a eu d'abord la jeune fille, nommée par sa mère poète en l'honneur de la Delphine de madame de Staël, et dont les poésies étaient signées Delphine Gay. Il y a eu ensuite une phase nouvelle, initiée par son mariage. Gautier (1857) écrit: «Madame Émile de Girardin ne date pour nous que de Napoline, un poème qu'elle publia en 1833, après son mariage» (p. vi). Et enfin, il y a l'époque glorieuse où elle emprunte le pseudonyme du vicomte de Launay pour écrire ses Chroniques parisiennes. Une telle fragmentation réduisait déjà les chances de Delphine de Girardin d'entrer dans les anthologies. Et de fait, le préfacier des Éditions des Femmes, Jean-Louis Vissière (1986), écrira en 1986: «Delphine Gay. Mme de Girardin. Le vicomte de Launay. Trois noms. Trois auteurs à succès. Une seule personne» (p. 7). En conséquence, la place que tenaient Delphine de Girardin et ses œuvres à leur époque nous échappe. Plusieurs de ces textes, et surtout les Chroniques parisiennes, semblent naître d'une plume contemporaine, dans l'apaisement passager du tourbillon que représente la vie dans une grande ville, d'où leur intérêt pour nous et nos étudiants. Par ailleurs, un roman comme La Canne de M. de Balzac propose une représentation originale, humoristique et vive du personnage qu'était Balzac. Autrement dit, l'œuvre de Delphine de Girardin répond au critère de pertinence dont on tente ici de dessiner quelques paramètres. Les jugements de Gautier et de Lamartine sur son œuvre sont élogieux, mais ce n'est pas sans à propos que Gautier (1857) explique pourquoi telle de ses œuvres n'a pas eu la réception qu'elle méritait:

Nous sommes surpris que Napoline n'ait pas eu un plus grand retentissement; il est vrai qu'alors avait lieu cette éclosion simultanée et magnifique de chefs-d'œuvre [...] et, au milieu 
de ce bouquet, éclatant avec un fracas lumineux, cette bombe à pluie d'argent fut moins remarquée qu'elle ne le serait aujourd'hui dans notre ciel vide et noir. (p. vi)

L'œuvre de Girardin s'inscrit, non pas dans une littérature des marges, d'où elle interpellerait les tenants du discours dominant. Elle participe, plutôt, de ce discours dominant mais le poids de ses contemporains a fait passer son œuvre du côté de l'impensé, qui est évacué par le binarisme qui maintient l'opposition entre la littérature canonique et la littérature marginale. ${ }^{6}$ Il faut donc cesser d'opposer le canon, un corpus restreint d'œuvres dotées a posteriori d'un caractère d'universalité et de valeur intrinsèque, à l'ensemble de la production qui se caractériserait tantôt par la reproduction de topö̈ et de procédés prévisibles, tantôt par la conformité idéologique, tantôt par le particularisme ou le circonstanciel. Il faut plutôt reconstituer la dimension dialogique de la littérature, recréer les échanges qui, en se nourrissant les uns les autres, contribuent à l'évolution de la pensée et de l'esthétique d'une époque donnée. Pour reconstituer la plurilogie de la vie littéraire de chaque époque étudiée, il faut mettre en présence les textes qui se répondent. Je veux enseigner Les Précieuses Ridicules? Ne devrais-je pas aussi enseigner Madeleine de Scudéry, Madame de Villedieu ou Marie de Gournay? Que nous apprendrait une comparaison entre les Lettres péruviennes (Madame de Graffigny) et les Lettres persanes (Montesquieu)? Indiana ne constitue-t-elle pas une version moins démoralisante d'Emma Bovary? Ourika, premier personnage noir s'exprimant à la première personne dans la littérature française, et créé par Claire de Duras, n'a-t-il pas autant à offrir que l'Atala de Chateaubriand?

Mais comment retrouver les textes a-canoniques des romancières (le mot apparaît, selon Le Robert, en 1844, symptôme d'un début d'exclusion des œuvres de femmes), dont presque la totalité de la production a été reléguée dans la littérature populaire, entraînant là, puis aux oubliettes, une part importante du roman historique, le roman psychologique et aussi le roman du quotidien? Par un lent travail de récupération, de réédition, de mise en ligne, qui fera remonter à la surface ces «cargaisons de volumes» (Lescure) qui s'effritent lentement à la Bibliothèque Nationale. L'inclusion de quelques femmes auteurs 
dans nos programmes permettrait de rendre compte, aussi, en ce qui concerne le XIXe siècle, et pour ne donner que quelques exemples, de la profondeur de leur pensée (Comtesse de Salm-Dyck, Marie d'Agoult, et surtout Mme de Staël, dont on lit plus que Corinne); ou bien, de reconstituer l'histoire du genre romanesque, qui doit beaucoup aux femmes (Sophie Cottin, Félicité de Genlis, André Léo); ou encore, de présenter des structures narratives, des parti-pris stylistiques, des points de vue thématiques offrant des variantes significatives au scénario lukacsien du héros en butte à la société (Isabelle de Charrière, Juliane de Krüdener, Claire de Duras); de révéler, aussi, l'évolution de genres littéraires très pratiqués mais peu étudiés comme la nouvelle (Sophie d'Arbouville), les recueils de pensées (Élisabeth de Roumanie), l'essai sur l'éducation (Anaïs de Bassanville), la correspondance (Eugénie de Guérin); de rendre justice, enfin, à des auteures résolument innovatrices, comme Judith Gautier et Rachilde.

Le problème ne se pose pas autrement en ce qui concerne la poésie: Planté (1998) affirme avec justesse, dans l'«Introduction» de son anthologie des femmes poètes du XIXe siècle, que les femmes ont publié de nombreux recueils qui ont fait l'objet d'une réception critique et académique favorable, et que plusieurs ont reçu le soutien des Baudelaire, Rimbaud, Verlaine, Mallarmé, Laforgue. Elle affirme:

Un tel écart entre la production poétique féminine et ce qui en a été transmis à la postérité, cette rapide invisibilisation viennent de ce que ces poèmes ont été, à un moment donné, jugés indignes de mémoire par les multipes instances qui décident de la reconnaissance littéraire, de la constitution et de la transmission du patrimoine poétique national. (p. iv)

Elle ajoute que la réinscription des femmes dans l'histoire de la poésie met en lumière le caractère arbitraire des périodisations. On peut ajouter que la définition des mouvements littéraires pourrait aussi être revue en fonction d'une inclusion des auteurs qui ont été marginalisés par les définitions a posteriori de ces mouvements. On a révisé la définition du Romantisme pour inclure les Petits Romantiques tels que Philothée O’Neddy, Hégésippe Moreau, Aloysius Bertrand; pourquoi ne pas la 
revoir afin d'inclure, non seulement Marceline Desbordes-Valmore, Élisa Mercoeur, mais aussi Louise Colet?

\section{LA MISE EN LIGNE DES TEXTES LITTÉRAIRES}

La tâche en somme, est de «reconstruire», comme dit Bourdieu (1998), «l'histoire du travail historique de déshistoricisation» (p. 90). Ce qui se joue, ici, est un déracinement de notre expérience littéraire, et ce «nous» inclut les étudiants: déracinement par rapport à l'époque étudiée, et aussi par rapport à notre époque. Dans ce contexte, Internet aura une importance déterminante pour l'avenir de l'enseignement de la littérature. Grâce à la toile, nous pouvons moderniser nos approches, et surtout avoir accès à une infinité de textes qui jusqu'ici étaient inaccessibles dans la plupart des institutions canadiennes. Mais malheureusement les serveurs déjà canoniques qui nous procurent des textes littéraires semblent voués à la reproduction du canon. La plupart des textes qu'on met en ligne à l'heure actuelle se trouvent déjà dans toute bibliothèque qui se respecte. L'importance de rendre disponible le plus grand nombre possible de textes aux fins de la recherche n'explique pas que les textes de femmes ne comptent actuellement que pour deux à six pour cent, selon les sites, des textes électroniques. Ce qui est frappant, c'est la constance de ce petit pourcentage, dans des sites consacrés aux classiques, comme Gallica classique ou Florilège, mais aussi dans des sites se donnant pour mandat la mise en ligne de textes inédits, comme celui de la Bibliothèque de Lisieux.

Le site de Gallica XIXe siècle (gallica.bnf.fr), qui regroupe aussi bien des textes documentaires portant sur l'éducation, la morale, la science et la médecine, que des textes littéraires, n'offre que 35 textes de femmes sur 1215, soit 2,8\%.' Le site de l'Ambassade de France (www.ambafrance.org/FLORILEGE) ne recense qu'une femme pour cinquante auteurs au XIXe siècle. Le site du projet ARTFL (www.lib.uchicago.edu/efts/ARTFL) compte à l'heure actuelle 8 femmes parmi 120 auteurs, toujours pour le XIXe siècle, soit une proportion de $6,6 \%{ }^{8}$ C'est sans doute du site de Lisieux (www.bmlisieux.com) que l'on pouvait s'attendre à mieux, étant donné qu'il est consacré à la mise 
en ligne de textes rares, épuisés et inédits. Compte tenu des critères de sélection établis par le webmestre (Olivier Bogros) du site de Lisieux (œuvres du domaine public, courtes, issues des collections de Lisieux ou de collections privées; équilibre entre les ouvres de fiction et les textes documentaires; complémentarité avec les autres bibliothèques virtuelles), on s'attendrait à une meilleure représentation des femmes auteurs sur ce site, qui pourtant ne signent que le même six pour cent des textes mis en ligne à ce jour. Ce plafonnement rend d'autant plus frappante la disproportion des efforts pour une meilleure accessibilité des textes.

On peut sans doute attribuer une partie de cette lacune à une certaine résistance des femmes face aux nouvelles technologies, résistance que l'on peut mettre en rapport avec un contexte socio-institutionnel moins propice à leur familiarisation avec ces technologies. Vu en termes de pouvoir, comme le propose Dale Spender (1996), Internet est territoire masculin (p. 166), un territoire dont les femmes doivent s'approprier les outils d'exploration. Et certaines initiatives ont déjà permis la mise en ligne de textes de femmes. Mais il est crucial que les responsables des entreprises d'envergure prennent en compte la nécessité de numériser de façon prioritaire les textes introuvables. Notons aussi que la mise en ligne des textes littéraires des régions francophones des Antilles et d'Afrique n'est possible que dans la mesure où ces régions auront accès aux équipements technologiques requis.

Il faut aussi intégrer la navigation à l'enseignement. S'il est vrai que le monde virtuel reproduit le monde réel, et par là ses structures de pouvoir, il permet aussi une révision globale des concepts d'apprentissage et d'enseignement. Non seulement pouvons-nous faire usage des ressources multimédia en classe, et rendre nos étudiants plus autonomes en encourageant les recherches électroniques qui les rendront plus compétents dans leur utilisation des nouvelles technologies; nous pouvons aussi repenser nos cours en utilisant l'hyperlien comme métaphore: un format de cours où la saisie des données n'est plus assujettie à des périodes strictes, mais commandée, plutôt, par les rapports qui surgissent à l'évocation d'un mot, d'un nom, d'un titre, d'un vers, d'une date. Les étudiants peuvent contribuer plus activement à 
leurs apprentissages et la navigation rend plus probable la création de liens/rapports intertextuels. Il est à prévoir que le modèle que nous imposent les nouvelles technologies, dans lequel la tabularité prend préséance sur la linéarité, nous amènera éventuellement à privilégier l'intertextualité aux dépens de la linéarité chronologique. Comme le dit Said (1999), «plus une œuvre est reliée à d'autres œuvres et d'autres histoires, plus précaires sont nos découvertes et plus stimulant est le partage de cette recherche avec les étudiants» (p. 3).

\section{QUELQUES MOTS POUR CONCLURE}

Toute la question, bien sûr, est de choisir des objectifs. En nous limitant au corpus des "grands auteurs,» pouvons-nous prétendre tenir compte des besoins, des intérêts et des objectifs de notre époque, de nos étudiants, et de nous-mêmes, pour reprendre les termes de Barbara Herrnstein Smith (1988):

What we speak of as a subject's 'needs,' 'interests,' and 'purposes' are not only always changing, but they are also not altogether independent of or prior to the entities that satisfy or implement them; that is, entities also produce the needs and interests they satisfy and evoke the purposes they implement. (p. 32)

Afin de sortir de cette boucle, il convient de se poser des questions qui mèneront à une révision de nos objectifs d'enseignement. Quels buts poursuivons-nous dans notre enseignement de la littérature classique? Donner un vernis de culture à nos étudiants, ou les initier à quelques approches critiques? Relever leur niveau de langue, ou les former à l'analyse stylistique? Leur faire connaitre les grands auteurs, ou leur donner un aperçu des pratiques textuelles d'une époque donnée? Selon les réponses que l'on apporte à ces questions, et même si on répond «un peu de tout,» les découpages et les sélections traditionnels paraissent de moins en moins adéquats. Entre les cours qui se consacrent à une problématique plus «spécialisée» et ceux qui adhèrent à des choix plus canoniques, il y a une troisième voie, qui accorde une place plus importante aux œuvres qui reflètent la pensée esthétique de leur époque, mais rendent compte de préoccupations trouvant encore un écho dans les 
aspirations de nos étudiants. C'est ce que j'appelle le critère de pertinence, qui se définit à partir de l'analyse de trois aspects d'une œuvre prise comme objet relationnel: ancrage d'une cuvre dans sa propre époque d'abord, c'est-à-dire dans le contexte où elle apparaît, rapports, ensuite, de cette œuvre à notre époque, et enfin, surtout, rapports dialogiques ou intertextuels avec des textes de multiples autres provenances, puisque l'établissement de réseaux intertextuels, qui devrait être facilité de manière exponentielle par la numérisation des textes, constitue le but ultime de la connaissance et de l'enseignement de la littérature, comme l'affirme Jean Larose (1991):

Parce que la réalité n'est pas ce qui est vu, senti, entendu et perçu, mais l'ensemble des rapports qui lient toutes ces choses entre elles et avec les souvenirs, pour faire connaitre la réalité à un élève, l'école de la littérature dépasse toute les autres. (p. 17)

\section{Les Notes}

1 Notre traduction.

2 Voir à ce sujet les travaux, figurant dans la bibliographie, de Herrnstein Smith, Lafarge, et Bourdieu (1977).

3 Bien que ces descripteurs aient été recueillis dans les annuaires et les sites Internet des universités concernées, il ne paraît pas utile de préciser ici leur origine spécifique. Par ailleurs, nous avons restreint l'étude aux universités anglophones, qui s'inscrivent dans une tradition commune, et qui sont assujetties aux mêmes contraintes reliées au niveau de connaissance de la langue de leurs étudiants.

4 La personne qui enseigne le cours «Barricades, bordels et champs de bataille» m'informe qu'elle met au programme Notre-Dame de Paris et Cyrano de Bergerac.

5 Sauf qu'on se serait attendu à trouver Stendhal dans le premier groupe et Vigny dans le second...

6 On maintient cette opposition tout en affirmant que la littérature canonique commence toujours par être marginale, que c'est son aspect 
innovateur, d'abord déstabilisant, qui lui permet de se distinguer. On trouve ce postulat chez Bloom.

7 Ces chiffres varient constamment, puisque des textes s'ajoutent tous les jours, mais la tendance n'a pas varié depuis un an.

8 Signalons toutefois que sept ou huit textes de trois femmes auteurs du XIXe siècle seront ajoutés sous peu à ce catalogue.

\section{Bibliographie}

Angenot, M. (1997). Les idéologies du ressentiment. Montréal, QC: XYZ Éditeur.

Barthes, R. (1984). Le bruissement de la langue. Essais critiques IV. Paris: Seuil, coll. «Points.»

Bloom, H. (1994) The western canon. New York, NY: Harcourt, Brace \& Company.

Bourdieu, P. (1977). La production de la croyance. Actes de la recherche en sciences sociales, 13 (février 1977), 3-43.

Bourdieu, P. (1998). La Domination masculine. Paris: Seuil.

Clark, T. (1997). The theory of inspiration. Manchester, UK: Manchester University Press.

Coste, D. (1989). Pour une histoire littéraire négative du XIXe siècle français. Les Genres de l'hénaurme siècle. Ann Arbor, MI: University of Michigan Press, 15-32.

Culler, J. (1997). Literary theory. A very short introduction. Oxford, UK: Oxford University Press.

Dubois, J. (1978). L'Institution de la littérature. Paris/Bruxelles: Nathan / Labor.

Eagleton, T. (1983). Literary theory. An introduction. Minneapolis, MN: University of Minnesota Press.

Fleming, B.E. (2000). What is the value of literary studies? New Literary History, 31(3), (Summer 2000), 459-486.

Foucault, M. (1969). Qu'est-ce qu'un auteur? Bulletin de la société française de philosophie, LXIV, 75-95.

Gautier, T. (1857). Madame Émile de Girardin. Oeuvres complètes de madame Émile de Girardin. Paris: Plon. 
Guillory, J. (1993). Cultural capital. The problem of literary canon formation. Chicago, IL: Chicago University Press.

Herrnstein Smith, B. (1988). Contingencies of value. Cambridge, MA.: Harvard University Press.

Lafarge, C. (1983). La valeur littéraire. Paris: Fayard.

Larose, J. (1991). L'amour du pauvre. Montréal, QC: Boréal.

Lescure, M. (1880). Notice. Madame de Genlis, Mademoiselle de Clermont. Paris: Librairie des Bibliophiles.

Miller, N.K. (1990). Feminist writing and the history of the novel. In M. Spilka \& C. McCracken-Flesher (Eds.), Why the novel matters; A postmodern perple (pp. 328-339). Bloomington, IN: Indiana University Press.

Mozet, N. (1992). À l'épreuve du féminin: littérature et mixité. Romantisme, 77, 3-7.

Planté, C. (1998). Introduction. Femmes poètes du XIXe siècle; Une anthologie. Lyon: Presses Universitaires de Lyon.

Ross, T. (1996). After the canon wars. Dalhousie Review, 76(3), 432-443.

Said, E.W. (1999). An unresolved paradox. MLA Newsletter, 3l(2), 3.

Sauvé, R. (2000). De l'éloge à l'exclusion. Les femmes auteurs et leurs préfaciers au XIXe siècle. Vincennes: Presses Universitaires de Vincennes.

Slama, B. (1992). Un chantier est ouvert.... Notes sur un inventaire des textes de femmes du XIXe siècle. Romantisme, 77, 87-94.

Spender, D. (1996). Nattering on the net: Women, power and cyberspace. Toronto, ON: Garamond Press.

Vissière, J.-L. (1986). Préface. Delphine de Girardin, Chroniques parisiennes 1836-1848. Paris: Éditions des Femmes. 